\title{
An Investigation of Reliability of the Sunderland Tracheosophageal Voice Perceptual Scale.
}

\author{
Coffey, MM
}

http://hdl.handle.net/10026.1/13201

\subsection{9/000493751}

Folia Phoniatrica et Logopaedica

Karger Publishers

All content in PEARL is protected by copyright law. Author manuscripts are made available in accordance with publisher policies. Please cite only the published version using the details provided on the item record or document. In the absence of an open licence (e.g. Creative Commons), permissions for further reuse of content should be sought from the publisher or author. 
Reliability of the SToPS

2 An investigation of reliability of the Sunderland Tracheosophageal Voice 3 Perceptual Scale

4

5

6 
Reliability of the SToPS

\section{Abstract}

3

4 Introduction: The consensus on how to effectively evaluate alaryngeal voice

5 outcomes remains limited. The Sunderland Tracheosophageal Voice Perceptual

6 scale (SToPS) was developed as a perceptual rating scale specifically for

7 tracheosophageal voice ${ }^{1}$. Currently, it is the only tracheosophageal voice specific

8 perceptual scale available and aims to address the limitations of previous scales.

9

10 Objective: To investigate inter rater reliability of the Sunderland

11 Tracheosophageal Voice Perceptual Scale when analysing alaryngeal voice across

12 a range of voice prostheses.

13

14 Methods: Prospective evaluation of inter rater reliability of the SToPS based on

15 audio recordings of 230 voice samples from 41 laryngectomy patients rated by 3

16 experts. Interval data was analysed using Intraclass Correlation Coefficients (ICC)

17 while categorical data was analysed using Kappa.

18

19 Results: ICC of above 0.6 was observed between raters for each prosthesis on a 20 majority of parameters demonstrating a good level of reliability. Reliability was

21 fair (ICC of between 0.40-0.59) on Q11 (Articulatory precision) and Q12

22 (Paralinguistics). Reliability was also fair (0.21-0.40) or slight (0.00-0.20) for Q2

23 (Tonicity), which was analysed using Kappa. Kappa of above 0.61 signified a good

24 level of reliability. 
Reliability of the SToPS

2 Conclusions: This study demonstrates good rater reliability for the majority of

3 parameters on the SToPS scale, supporting the use of this tool within the clinical

4 realm. However further research is required to ascertain if any methods of

5 increasing inter rater reliability on those parameters which did not reach good

6 reliability can be identified.

$7 \quad$ Level of evidence: $2 b$ Individual cohort study

8

9

10 
Reliability of the SToPS

\section{2}

3

4

\section{Evaluation of post laryngectomy voice}

\section{Introduction}

Laryngectomy involves the removal of the larynx in its entirety, usually as a treatment for advanced laryngeal cancer. As a consequence, this surgery profoundly affects the ability to communicate. The gold standard for communication rehabilitation after laryngectomy is surgical voice restoration (SVR) ${ }^{2}{ }^{3}$ also known as tracheosophageal voice. This technique involves the placement of a one way valved voice prosthesis in a puncture between the trachea and oesophagus 45 . The voice prosthesis shunts lung air from the oesophagus to a vibratory segment within the reconstructed throat to produce tracheosophageal voice. The ultimate objective of SVR is to provide the patient with the optimal voice possible without a larynx ${ }^{1}$. However, consensus on the most appropriate measure of voice outcome post laryngectomy is lacking..

Although most of the empirical research concerning laryngeal voice has focused on acoustic measures of frequency, intensity and duration, these measures do not necessarily indicate how well an individual communicates in a social situation. Auditory perceptual rating involves an expert listener judging a voice sample according to different parameters ${ }^{6}$ which may include intelligibility, voice quality and acceptability 7. Auditory perceptual evaluation of tracheosophageal voice quality has been posited as the most valid measure of SVR outcome ${ }^{1}$. There are a number of well-established voice quality rating scales which provide perceptual parameters for the patients with a larynx including the Buffalo Voice Profile ${ }^{8}$, the Vocal Profile Analysis Scheme ${ }^{9}$, Grade, Roughness, Breathiness, Asthenia, Strain 
Reliability of the SToPS

2 (GRBAS) scale ${ }^{10}$, and Consensus Auditory Perceptual Evaluation of Voice ${ }^{11}$. Of

3 these, the strongest validity and reliability has been established for the GRBAS

4 12,13. The GRBAS has been used to assess auditory perceptual aspects of

5 tracheosophageal voice in several studies 14-16 17,18. However, use of the GRBAS to

6 measure perceptual aspects of tracheosophageal voice has been considered

7 suboptimal due to the fundamental differences in tracheosophageal and laryngeal

8 voice ${ }^{1}$. As the phonatory source of alaryngeal voice (vibratory segment) contrasts

9 significantly with that of laryngeal voice (vocal folds), the use of a rating scale

10 validated for the latter population poses limitations for post laryngectomy

11 patients. Additionally, some perceptual features of alaryngeal voice such as tone

12 and extraneous noise when covering the stoma to produce voice are unique and

13 central to tracheosophageal voice quality and are not included in the GRBAS scale.

14 Critically, studies which have used the GRBAS ${ }^{14-18}$ or other perceptual scales 19,20

15 have failed to specify an anchor baseline so it is unclear whether raters have

16 compared voice stimuli to that of normal laryngeal voice or optimal 17 tracheosophageal voice.

19 STOPS

20 The Sunderland Tracheosophageal Voice Perceptual scale (SToPS) was developed

21 as a perceptual rating scale specifically for tracheosophageal voice ${ }^{1}$. Currently, it

22 is the only tracheosophageal voice specific perceptual scale available. The $\underline{\text { SToPS }}$

23 was developed as means of overcoming the major conceptual and methodological

24 problems inherent in other studies of tracheosophageal voice, such as poorly

25 defined terminology and impressionistic vocabulary ${ }^{21}$. The STOPS includes

26 specific and clear guidance to define terminology used for each parameter. In 
Reliability of the SToPS

2 addition, the $\underline{\text { SToPS }}$ crucially defines the anchor baseline for parameters as

3 optimal tracheosophageal voice rather than normal laryngeal voice.

4

5

6 Reliability

7

8 Measurement is a way of understanding, evaluating and differentiating

9 characteristics of people and objects ${ }^{22}$ and forms the basis for making decisions

10 or drawing conclusions in scientific research. A crucial prerequisite for clinical

11 measurement is reliability. Reliability indicates the consistency and lack of errors

12 in a tool ${ }^{22,23}$. As the ability to simply produce voice with a prosthesis following

13 SVR is unlikely to be sufficient indication of functional ability to communicate in

14 everyday situations, it is of clinical relevance to investigate the reliability of the

$15 \underline{\text { SToPS. }}$ As intra rater reliability for expert raters had previously been established

16 as good or above for all parameters of the $\underline{\text { SToPS }}$ except for accent, reading ability

17 and articulatory precision ${ }^{24}$, this study focuses on the investigation of inter rater 18 reliability.

19

20 Aim

21 To investigate inter rater reliability of the Sunderland Tracheosophageal Voice

22 Perceptual Scale

23 Hypothesis

24 Experts will not achieve a good level of inter rater reliability when they use the

25 SToPS to rate alaryngeal voice. Should a good level of inter rater reliability be

26 achieved, this will support the clinical relevance for the SToPS in identifying

27 functional tracheosophageal voice for patients post laryngectomy. 
Reliability of the SToPS

Methods

5

SToPS

The Sunderland Tracheosophageal Voice Perceptual Scale (SToPS) for

9 professional raters was originally developed as a 14-item auditory perceptual

10 scale divided into two domains: (i) Six Voice quality parameters (perceptual voice

11 tonicity, strain, wetness, impairment of volume, impairment of social acceptability

12 of voice and whisper), and (ii) seven parameters not related to voice quality

13 (impression of intelligibility, stoma blast, impairment of fluency, impairment of

14 articulatory precision, positive features of articulation, accent and poor reader)

15 and an overall score voice rating. The scale later underwent item reduction and

16 now contains 10 parameters. Ref

17

18 Tone relates to the amount of pressure used to produce tracheosophageal voice.

19 The perceptual voice tonicity parameter is measured on an 11 point bipolar

20 semantic scale reflecting the continuum of tone ${ }^{25}$ from hypotonic (too little tone)

21 to hypertonic (too much tone) ${ }^{1}$. As stenotic voice occurs only in the absence of

22 tone it is measured with a separate arm to the tone scale ${ }^{1}$. As stenosis is either

23 present or absent, it is not rated along a graded continuum. For each individual

24 voice sample, only one arm of the scale is chosen by a rater. Each of the remaining

255 items in the voice quality parameters domain are measured on a 4 point equally

26 appearing interval scale 0 (optimal tracheosophageal voice quality), 1 (mild), 2

27 (moderate) and 3 (severe). 
Reliability of the SToPS

2 Each of the parameters not related to voice quality, with the exception of positive

3 features of articulation is measured on a 4 point equally appearing interval scale

40 (optimal tracheosophageal voice quality), 1 (mild), 2 (moderate) and 3 (severe).

5 Positive features of articulation are measured on an alternatively worded 4 point

6 equally appearing interval scale 0 (neutral), 1 (good), 3 (excellent), and 4

7 (outstanding).

8

9 The parameter 'overall grade" is measured using a four point interval scale $0=$

10 Excellent; 1 = Good; 2 = Adequate; 3 = Poor. This design is similar to the GRBAS

11 scale 10 except that the value 0 represents optimal tracheosophageal voice quality

12 as opposed to "normal" laryngeal voice quality.

Raters

16 Three Speech and Language Therapy raters were chosen. Each rater had at least 17 five years experience specialising in the rehabilitation of communication post 18 laryngectomy and other head and neck cancer patients and had completed 19 advanced training in the field.

21 Training of raters

22

23 Each rater participated in three hours of training with the investigator in the use

24 of the SToPS. This training took place during two conference calls of 90 minutes

25 length and included practice ratings of ten anonymised audio samples of 26 laryngectomy participants reading the Rainbow Passage. During training queries 
Reliability of the SToPS

2 about individual items on the SToPS scale were raised. These parameters were

3 discussed with the main author of the SToPS. Clarifications provided were passed

4 onto all three raters regardless of how many raters had initially raised a query.

5 
Reliability of the SToPS

\section{Voice stimuli}

230 voice samples were elicited from 41 post laryngectomy participants. Please see table 1 for demographic details. Participants were recruited from the outpatient caseload of Head and Neck cancer patients at a large centre in Exclusion criteria included participants without a voice prosthesis, less than 3 months post surgery or post operative oncological treatment. Each participant trialled up to 6 randomised voice prostheses over 2 appointments within a 72 hour period. Participants were blinded to prosthesis type and a voice sample was provided for each for each prosthesis. This data was used in a subsequent study investigating the differences between voice prostheses in terms of voice outcome.

For each prosthesis trial, participants had a Speedlink SL-8691-SBK spes clip on metal microphone (Speedlink, Weertzen, Germany) attached to their clothing 10 $\mathrm{cm}$ lateral to the stoma on the opposite side to the hand used to occlude the stoma during voicing. All subjects produced voice by occluding their stoma rather than depressing a humidification exchange device or using a hands free attachment. Subjects read a short version of the Rainbow passage ${ }^{26}$, (see appendix). This was recorded onto a Sony ICD-PX820 Digital Voice Recorder with flash 2 GB (Sony, Weybridge, UK) in MP3 format to be rated later by experts.

\section{Data analysis}

Recordings of voice samples with individual voice prostheses were extracted in MP3 format and transferred to Final Cut Pro (Apple, California, USA) to allow titles to be added to indicate anonymised subject number and anonymised voice prosthesis letter. Voice samples were then exported to 3 Verbatim 4GB pinstripe 
Reliability of the SToPS

2 USB memory sticks (Verbatim, Surrey, UK). Raters were blinded to subject,

3 prosthesis type, gender, type of laryngectomy surgery (extended laryngectomy or

4 standard total laryngectomy) and history of radiotherapy and chemotherapy.

5 Voice samples were posted to 3 expert Speech and Language Therapy raters along

6 with blank numbered and lettered SToPS forms which corresponded to each voice

$7 \quad$ sample for each subject.

8

\section{Statistical analysis}

11 Data was entered and analysed in IBM SPSS (Statistical Product and Service 12 Solutions) version 23 (IBM Armonk, New York). The $\underline{\text { SToPS }}$ consists of 14 13 parameters, 13 of which $(\mathrm{Q} 1, \mathrm{Q}-\mathrm{Q} 14)$, are rated from $0-3$ on an interval scale. $\underline{\mathrm{A}}$ 14 further parameter, Q2 of the SToPS is rated on an 11 point bipolar semantic scale 15 which yielded categorical data.

17 Intraclass correlation coefficients (ICC) were used to analyse reliability of interval 18 scale parameters. A 2 way mixed model was chosen as each subject was assessed 19 by the same set of raters who have been purposely and not randomly selected 27,28. $20 \quad 0.6$ ICC has previously been indicated as signifying a useful ${ }^{29}$ and good ${ }^{30}$ level of 21 reliability. ICC of between 0.40 and 0.59 has been defined as signifying a fair level 22 of reliability ${ }^{30}$. This interpretation was used to benchmark inter rater reliability 23 interval level data.

24 Cohen's kappa was used to analyse reliability of categorical data extracted from 25 Q2 (Perceptual Tonicity - amount of pressure used to produce tracheosophageal 
Reliability of the SToPS

2 voice) on the SToPS scale. In order to examine inter rater reliability for Q2, data

3 were recoded into 4 categories as follows:

4 Hypotonic 5, 4, 3, 2, 1 was recoded as 1

- $\quad$ Tonic 0 was recoded as 2

- $\quad$ Hypertonic 5, 4, 3, 2, 1 was recoded as 3

- $\underline{\text { Stenosis } 5 \text { was recoded as } 4}$

$\underline{\text { Reliability was calculated using kappa to see whether raters agreed } 2 \times 2}$

- $\quad$ Rater 1x Rater 2

- $\quad$ Rater 1x Rater 3

- $\quad$ Rater 2 x Rater 3

12 Analysis was conducted for reliability by prosthesis type by splitting data by

13 prosthesis type and then using cross tabs for kappa analysis by rater $2 \times 2$.

14 The Landis and Koch ${ }^{31}$ classification of 0.61 as a good level of reliability, $0.41-0.60$

15 as moderate reliability $0.21-0.40$ as fair reliability and $0.00-0.20$ as slight

16 reliability was used to analyse categorical level data.

\section{Results}

21 The majority of parameters $(\mathrm{Q} 1, \mathrm{Q} 3, \mathrm{Q} 5, \mathrm{Q} 7, \mathrm{Q} 8, \mathrm{Q} 9, \mathrm{Q} 13, \mathrm{Q} 14)$ reached an ICC of 0.60

22 indicating a good level of reliability (table $\underline{2}$ ). Parameters, which did not reach an

23 ICC of 0.60 are highlighted in greyscale. While reliability was not observed on Q4

24 ("Wetness" of voice quality) for the Blom Singer Low pressure voice prosthesis

25 nor on Q10 (Impairment of fluency) for the Blom Singer Duckbill voice prosthesis,

26 the ICC for both prostheses on both parameters approached good reliability.

27 Reliability for Q11 (Impairment of articulatory precision) was fair as opposed to 
Reliability of the SToPS

2 good except for the low-pressure prosthesis. Reliability was reached amongst

3 raters for only three of the voice prostheses (Blom Singer Duckbill, Blom Singer

4 Low pressure and Provox NID) but was fair for other prostheses on Q12 (Positive

5 features of articulation - paralinguistics/diction).

6

7

\section{Reliability of Q2 Bipolar Semantic Scale data from STOPS}

Results of this analysis are outlined in table 2.1, 2.2 and 2.3

Reliability between raters was therefore only fair or slight for Q2 Tonicity across voice prostheses.

\section{Discussion}

\section{Expert raters inter rater reliability on the SToPS}

Reliability was investigated to ascertain whether there was a good level of agreement among all three raters when using the SToPS to perceptually judge voice. Parameters with poor reliability were Q2 - Perceptual Voice Tonicity, Q11Impairment of articulatory precision and Q12 - Positive features of articulation (paralinguistics/diction). Q2 relates to tonicity of the vibratory segment or the amount of pressure used to produce alaryngeal voice. Clinically, a patient with a tonic voice will be able to produce fluent sound of adequate intensity without effort. A tonic voice has been defined as the ability to sustain /a:/ for 10 seconds and produce 10-15 syllables per breath ${ }^{32}$ or to sustain /a:/ 8 seconds and count from 1-15 on one breath ${ }^{33}$. A previous study ${ }^{1}$ examined inter rater agreement between 12 Speech and Language Therapists and 10 ENT surgeons for Q2 of the STOPS. While inter rater agreement was only moderate for the raters as a whole, 
Reliability of the SToPS

2 it was good for the subgroup of Speech and Language Therapists with specific

3 voice experience. Inter rater reliability was poor for three expert Speech and

4 Language Therapist raters in this study, each of whom had demonstrated a strong

5 understanding of tone within training sessions. The experience of Speech and

6 Language Therapists in this study was primarily in head and neck cancer rather

7 than specifically with laryngeal voice. This factor may account for the superior

8 agreement achieved on Q2 in a previous study ${ }^{1}$ However, the statistical

9 methodology which involved recoding data from Q2 from an 11 point equally

10 appearing interval scale into a four point categorical scale analysed with Kappa

11 may have been a further factor in the poor reliability found in this study. Recoding

12 data in this manner changes tonicity from a continuum to a categorical scale and

13 thus may alter analysis. The use of Cohen's Kappa for analysis is based on

14 absolute agreement. In examining a parameter such as tonicity, it may not be

15 possible to attain absolute agreement within hypertonic and hypotonic aspects of

16 the continuum. Both hypertonicity and hypotonicity contain a spectrum of

17 variety.

19 Similarly the complexity of the scale used to measure Q2 may have influenced

20 levels of reliability achieved.

22 Q11- Impairment of articulatory precision demonstrated fair rater reliability only.

23 This parameter measures the degree of the lack of precision or "slurring" in

24 speech. Lack of articulatory precision can be influenced by a number of factors

25 including fatigue and sometimes accent. During training of expert raters, Q11 was

26 not identified as one that needed further clarification. However, as the experience 
Reliability of the SToPS

2 of the expert raters involved in this study was predominantly with head and neck

3 cancer rather than with voice, it is possible that they were less familiar with the

4 defined baseline, which used the Vocal Profile Analysis scale as a reference. This

5 factor may have accounted for the fair rater reliability on this parameter. The final

6 parameter to demonstrate fair rater reliability was Q12 Positive features of

7 articulation (paralinguistics/diction). Positive features of articulation refer to

8 diction, intonation or pause features that have an overall positive effect but are

9 not part of the voice signal. Similarly to Q11, Q12 was not identified during

10 training as one that required further definition. Fair rater reliability on this

11 parameter and on Q11 may simply reflect the difficulties of assessing articulation

12 and diction in laryngectomy patients, who present with an underlying disordered 13 voice.

15 This study examined the reliability of the $\underline{\text { SToPS }}$ across a range of voice prostheses

16 as part of the preparatory work for a later study examining differences between 17 prostheses in terms of voice quality. Some voice prostheses notably differed in 18 levels of reliability achieved on parameters $4,10,11$ and 12 of the STOPS. The 19 attributes of different types of prostheses may affect tracheoesophageal voice and 20 therefore results of auditory perceptual analysis. This is an area that may warrant 21 further research.

Measurement of reliability

25 This statistical methods used to analyse reliability in this study correspond with 26 those conventionally used for measurement of categorical data (Cohen's kappa) ${ }^{34}$ 
Reliability of the SToPS

222 and interval data (ICC) 2722 . As a previous study ${ }^{124}$ utilised weighted kappa to

3 evaluate reliability on all parameters of the STOPs, a possible limitation of this

4 study was the use of ICCs rather than kappa to measure interval data. ICCs have

5 been used extensively to measure reliability of pathological voice quality and for

6 this reason were utilised in this study. However, the use of ICC is largely based on

7 a framework of psychological testing. This framework substitutes listeners for test

8 items and voices for test subjects and implies that a new set of raters would

9 produce the same mean ratings for the same test voices. ${ }^{35}$. This approach has been

10 challenged as neither representing patterns of reliability nor overall agreement

11 for specific voice samples ${ }^{35}$. The alternative to ICC is weighted kappa. Weighted

12 kappa addresses the issue of Cohen's kappa failing to take into account the degree

13 of disagreement between raters by enabling greater weight to be assigned to some

14 rater disagreements than others ${ }^{36}$ However, kappa has been criticised as less

15 informative when used with more than 2 raters and analysing exact agreement

16 without accounting for "close" agreement ${ }^{22}$. In addition, with the use of kappa,

17 variance of subjects may be an issue, as a homogenous group of subjects is more

18 likely to show a high percentage of agreement, rather than a true reflection of

19 reliability ${ }^{22}$. The lack of consensus and limited evidence regarding the optimal

20 methodology to measure rater reliability in perceptual evaluation of both

21 laryngeal and alaryngeal voice supports the need for further research in this area.

\section{Conclusions}

24

25 This study investigated inter rater reliability of the Sunderland Tracheosophageal

26 Voice Perceptual Scale. The findings presented in this study supports the SToPS

27 as a reliable tool for the auditory perceptual rating of alaryngeal voice. However, 
Reliability of the SToPS

2 it is acknowledged that further research may be required to improve levels of

3 agreement for parameters related to tonicity, articulatory precision and positive

4 features of articulation.

5

6 


\section{References}

1. Hurren A, Hildreth A, Carding P. Can we perceptually rate alaryngeal voice? Developing the Sunderland Tracheoesophageal Voice Perceptual Scale. Clinical otolaryngology : official journal of ENT-UK ; official journal of Netherlands Society for Oto-Rhino-Laryngology \& Cervico-Facial Surgery 2009; 34:533-538.

2. Kazi R, Nutting C, Evans PR, Harrington K. A short perspective on the surgical restoration of alaryngeal speech. Southern medical journal 2009; 102.

3. Hancock K, Ward E, Lawson N, van As-Brooks CJ. A prospective, randomized comparative study of patient perceptions and preferences of two types of indwelling voice prostheses. Int J Lang Commun Disord 2012; 47:300-309.

4. Singer M, Blom E. An endoscopic technique for restoration of voice after laryngectomy. The Annals of otology, rhinology, and laryngology 1980; 89:529-533.

5. Blom E, Singer M. Surgical-Prosthetic Approaches for Post-laryngectomy Voice Restoration Laryngectomy Rehabilitation. Houston: College Hill Press, 1979.

6. Carding P, Carlson E, Epstein R, Mathieson L, Shewell C. Formal perceptual evaluation of voice quality in the United Kingdom. Logoped Phoniatr Vocol 2000; 25:133-138.

7. Doyle P, Eadie T. The perceptual nature of alaryngeal voice and speech. In: Doyle P, Keith R, eds. Contemporary Considerations In The Treatment And Rehabilitation Of Head And Neck Cancer: Voice, Speech, And Swallowing. Austin, Texas: Pro Ed, 2005:113-139.

8. Wilson D. Voice problems of children. Baltimore: Williams and Wilkins, 1987.

9. Laver J. The phonetic description of voice quality. Cambridge: Cambridge University Press, 1980.

10. Hirano M. Clinical Examination of Voice New York: Springer Verlag, 1981.

11. Kempster GB, Gerratt BR, Verdolini Abbott K, Barkmeier-Kraemer J, Hillman RE. Consensus auditory-perceptual evaluation of voice: development of a standardized clinical protocol. Am J Speech Lang Pathol 2009; 18:124-132.

12. De Bodt MS, Wuyts FL, Van de Heyning PH, Croux C. Test-retest study of the GRBAS scale: influence of experience and professional background on perceptual rating of voice quality. J Voice 1997; 11:74-80.

13. Wuyts FL, De Bodt MS, Van de Heyning PH. Is the reliability of a visual analog scale higher than an ordinal scale? An experiment with the GRBAS scale for the perceptual evaluation of dysphonia. Journal of Voice 1999; 13:508-517.

14. Omori K, Kojima H. Neoglottic vibration in tracheoesophageal shunt phonation. European archives of oto-rhino-laryngology : official journal of the European Federation of Oto-Rhino-Laryngological Societies (EUFOS) : affiliated with the German Society for Oto-Rhino-Laryngology - Head and Neck Surgery 1999; 256:501-505. 
15. Kazi R, Singh A, Mullan Get al. Can objective parameters derived from videofluroscopic assessment of post laryngectomy valved speech replace current subjective measures? An e tool based analysis. Clinical Otolaryngology 2006; 31:518.

16. Kazi R, Singh A, Venkitaraman R, Sayed S, RhysEvans P, Harrington K. Is electroglottography-based videostroboscopic assessment of postlaryngectomy prosthetic speech useful? Journal of Cancer Research and Therapeutics 2009; 5:85-92.

17. Kazi R, Kiverniti E, Prasad Vet al. Multidimensional assessment of female tracheoesophageal prosthetic speech. Clinical otolaryngology 2006; 31:511-517.

18. Schindler A, Mozzanica F, Ginocchio D, Invernizzi A, Peri A, Ottaviani F. Voice-related quality of life in patients after total and partial laryngectomy. Auris Nasus Larynx 2012; 39:77-83.

19. van As CJ, Hilgers FJ, Verdonck-de Leeuw IM, Koopmans-van Beinum F. Acoustical analysis and perceptual evaluation of tracheoesophageal prosthetic voice. Journal of voice 1998; 12:239.

20. Finizia C, Dotevall H, Lundstrom E, Lindstrom J. Acoustic and perceptual evaluation of voice and speech quality: a study of patients with laryngeal cancer treated with laryngectomy vs irradiation. Archives of otolaryngology--head \& neck surgery 1999; 125:157-163.

21. vanAs C. Tracheoesophageal speech: A multidimensional assessment of voice quality. Institute of Phonetic Sciences. Amsterdam: University of Amsterdam, 2001:199.

22. Portney L, Watkins M. Foundations of Clinical Research - Applications to practice. New Jersey: Pearson Education International, 2009.

23. Lachin JM. The role of measurement reliability in clinical trials. Clinical trials (London, England) 2004; 1:553-566.

24. Hurren A. The development of a new rating scale for the perceptual assessment of tracheoesophageal voice quality outcome following total laryngectomy Institute of Health and Society: Univeristy of Newcastle, 2014:307.

25. Perry A. Vocal rehabilitation after total laryngectomy Leicester School of Speech Pathology. Leicester: De Montfort University, 1989:176.

26. Fairbanks D. Voice and Articulation Drill book. New York: Harper \& Brothers, 1960.

27. Shrout $P$, Fleiss J. Intraclass correlation: Uses in assessing rater reliability. Psychological bulletin 1979; 86:420-428.

28. McGraw K, Wong S. Forming inferences about some intraclass correlation coefficients. . Psychol Methods 1996; 1:30-46.

29. Chinn S. Statistics in respiratory medicine. 2. Repeatability and method comparison. Thorax 1991; 46:454-456.

30. Cicchetti D. Guidelines, Criteria, and Rules of Thumb for Evaluating Normed and Standardized Assessment Instrument in Psychology. 1994.

31. Landis JR, Koch GG. The measurement of observer agreement for categorical data. Biometrics 1977; 33:159-174.

32. Lewin JS, Baugh RF, Baker SR. An objective method for prediction of tracheoesophageal speech production. J Speech Hear Disord 1987; 52:212-217. 
Reliability of the SToPS

2 33. Blom ED, Singer MI, Hamaker R. Tracheoesophageal voice restoration 3 following total laryngectomy. Singular Pub. Group, 1998.

4 34. Cohen J. A Coefficient of Agreement for Nominal Scales. Educational and

5 Psychological Measurement 1960; 20:37-46.

6 35. Gerratt BR, Kreiman J. Theoretical and methodological development in

36. Cohen J. Weighted kappa: nominal scale agreement with provision for the study of pathological voice quality. Journal of Phonetics 2000; 28:33570:213-220. 